\title{
Study Wetting Coal Dust Fraction of Particles of Surfactant Solutions
}

\author{
Ivan S. Elkin and Igor B. Istomin* \\ Kuzbass State Technical University \\ named after T.F. Gorbachev \\ 28 Vesennyaya Str., Kemerovo, 650000, Russia
}

Received 08.05.2016, received in revised form 09.07.2017, accepted 04.10.2017

The results of experimental studies and theoretical basis for wetting solutions surfactants coal particles of small size fractions. On the basis of a physical model on experimental experience shows wetting of the coal particles, resulting from pulverized coal lumps pulverized state to substantially change the structural and surface properties of the coal, which leads to a substantial change in the mechanical, physical and chemical properties of coal.

Keywords: coal, wetting agent, a surfactant, a fraction hydrotreating, filtering, moisturizing, coal seam, flotation coal enrichment.

\section{Исследование смачивания пылевидных \\ частиц фракций угля растворами ПАВ}

И.С. Елкин, И.Б. Истомин

Кузбасский государственный технический университет имени Т.Ф. Горбачева

Россия, 650000, Кемерово, ул. Весенняя, 28

\begin{abstract}
Представлены результаты экспериментальных исследований и теоретическое обоснование по смачиванию растворами поверхностно-активных веществ частии углей фракций малых размеров. На основе физической модели экспериментального опыта по смачиванию частии угля показано, что в результате измельчений угля от крупных кусков до пылеобразного состояния существенно изменяются структурные и поверхностные свойства угля, что приводит к существенному изменению механических, физико-химических свойств углей.
\end{abstract}

Ключевые слова: уголь, смачиватель, поверхностно-активное вещество, фракция, гидрообработка, фильтрация, увлажнение, угольный пласт, флотаџия, обогащение угля.

(c) Siberian Federal University. All rights reserved

* Corresponding author E-mail address: jelkin@mail.ru 
Одной из современных технологий, используемых при разработке угольных месторождений, является гидрообработка угольного пласта, где главным компонентом выступает вода. Эффективность ее зависит от смачивающей способности жидкости, межфазных процессов на границе раздела уголь-жидкость-газ $[1,2]$. С другой стороны, обеспыливание выработанного пространства, особенно в очистном забое при подземной разработке угольных месторождений, пылеподавление в производственном процессе признаны важной проблемой в применении современных технологий. Одно из направлений решения этой проблемы - поиск и использование наиболее эффективного поверхностно-активного вещества (ПАВ). В поисках технологических решений важной задачей становится теоретическое моделирование процессов смачивания и межфазных взаимодействий на границе уголь-жидкость-газ, позволяющее детально исследовать физико-химические процессы на границе раздела [3-7].

\section{Материалы и методы исследований}

Цель данных исследований - изучение процессов смачивания и возможности его интенсификации процесса при применении ПАВ, определение основных факторов, влияющих на эффективность смачивания углей.

Проблемой эффективного применения ПАВ является их выбор и определение оптимальных концентраций $[8,9]$. При существующем большом разнообразии методов, способов и методик для исследования эффективности ПАВ и межфазных взаимодействий нами был выбран один из наиболее известных методов, основанный на изучении скорости смачивания пылеобразных частиц на поверхности жидкости.

Сущность данных экспериментальных опытов исследований заключалась в следующем. В кювету с приготовленным раствором с известной концентрацией ПАВ или водой на поверхность жидкости распыляли, рассеивали известное количество исследуемой фракции угля или другого материала. С помощью секундомера измерялось время и количество осевших на дно кюветы частиц угля. Время проведения наблюдений в зависимости от скорости протекания смачивания составляло от 15 мин до нескольких часов, а в отдельных случаях несколько суток.

При использовании данного метода в лабораторных условиях проведены наблюдения на различных фракциях углей разных марок углей, пылеобразных частицах от промышленных до строительных материалов с размерами частиц $0,0-0,25,0,25-0,5,0,5-1,0$ мм и др., полученные путем механического дробления и последующего разделения на фракции с помощью стандартных лабораторных сит. В качестве ПАВ применяли современные смачиватели типа «Неолас», «Эльфор», «Заслон», СП-1 и др., широко используемые в угольной промышленности.

\section{Результаты}

Некоторые результаты исследований представлены на рис. 1 в виде графических зависимостей скорости оседания частиц на дно кюветы от времени $v=f(t)$, где в качестве ПАВ использован смачиватель «Неолас». Из результатов следует, что наиболее гидрофобными свойствами обладают фракции угля марки КЖ с размером частиц $0,0-0,25$ мм. Время их оседания для этой марки угля для воды составляет несколько суток. Существенными факторами, оказываю- 
щими значимое влияние на изменение скорости оседания, является вид ПАВ и его концентрация, температура воды, атмосферное давление и др.

На рис. 2 приведены зависимости количества осевших частиц за одну минуту от концентрации ПАВ в растворе, полученные в ходе исследований. Поверхностно-активные вещества существенно изменяют скорость оседания частиц.

Результаты экспериментов по смачиванию частиц различных размеров показывают, что для углей при изменении размеров частиц вследствие измельчения значительно увеличивается физико-химическая активность частиц. Из наблюдений за поведением частиц следует, что удельная поверхность меньших частиц значительно больше. Дробление частиц приводит к

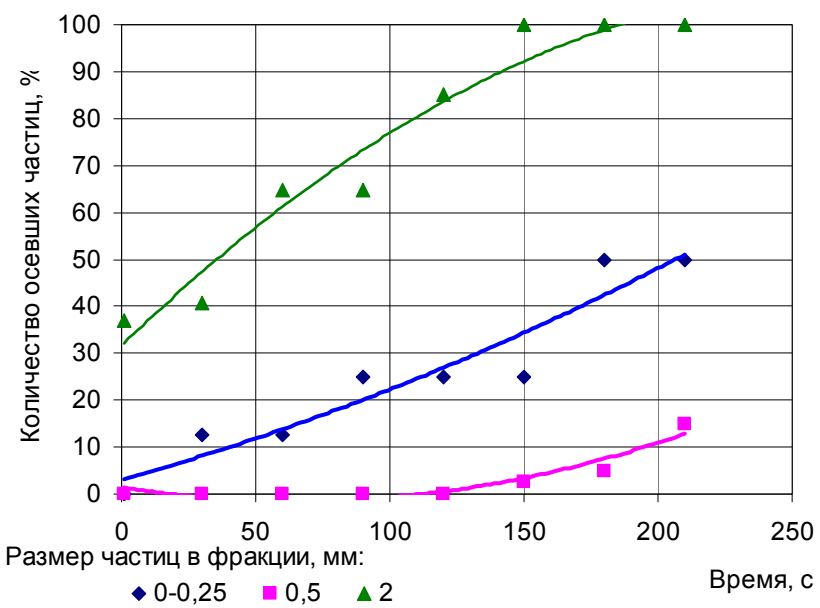

Рис. 1. Количество осевших частиц угля на дно кюветы в зависимости от времени

Fig. 1. The amount of settled coal particles at the bottom of the cuvette as a function of time

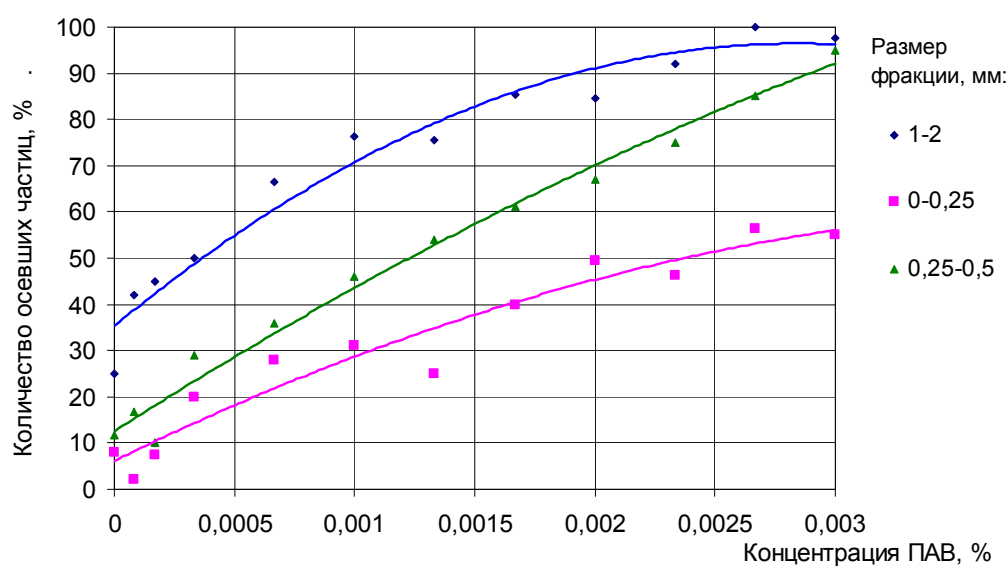

Рис. 2. Количество осевших частиц угля с течением времени в зависимости от концентрации ПАВ для частиц различных фракций

Fig. 2. The amount of settled coal particles over time as a function of From the concentration of surfactants for particles of different fractions 
разрушению закрытых капиллярных систем с образованием новой активной поверхности, как правило, обладающей гидрофобными свойствами. Общие закономерности здесь следующие:

- с увеличением массы частицы скорость оседания их растет;

- с уменьшением размера частиц повышается удельная площадь частицы и влияние межфазных взаимодействий на смачивание.

Результаты исследований показали, что процесс смачивания пылеобразных частиц сложен по своему механизму. При нем наблюдается несколько одновременно протекающих процессов и явлений: смачивание поверхности частицы, адсорбция, вытеснение с поверхности твердой фазы молекул газа, фильтрация жидкости по капиллярам частиц под действием лапласовских сил смачивания и др.

\section{Обсуждение результатов}

Интересна теоретическая сторона наблюдаемых процессов - модель описания процесса смачивания поверхности частиц малых размеров растворами ПАВ. Для обоснования этих процессов была разработана физическая модель смачивания частиц. Согласно предлагаемой модели угли являются капиллярно-пористыми телами с высокоразвитыми системами пор и капилляров как закрытого, так и открытого типа, в которых содержится главным образом газовая и жидкая фаза в свободном и адсорбированном состояниях $[3,7]$. По мере смачивания поверхности и заполнения капилляров жидкостью одновременно происходит вытеснение адсорбированного газа с поверхности частицы и сжатие воздуха внутри капилляров частиц жидкостью вследствие фильтрации под действием в основном лапласовских сил смачивания. При этом сила Архимеда $F_{\text {А }}$ остаётся неизменной, а масса частицы $m$ и сила тяжести $F_{m}$ увеличиваются. Нарушается равновесие между силами, и частица погружается, опускается на дно кюветы (рис. 3).

Рассматривая межфазные взаимодействия на границе раздела, будем считать, что процесс смачивания заключается в проникновении смачивающей жидкости внутрь частицы по капиллярной системе под действием лапласовских сил, вытесняя газ из капиллярной системы частицы, если капилляры насквозь пересекают частицу, или сжатие газа внутри капилляров происходит под действием лапласовских сил, если капилляры имеют только один канал входа.

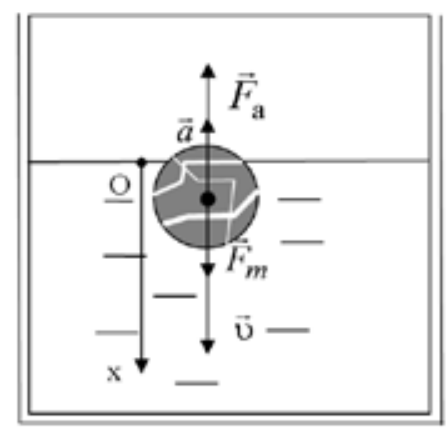

Рис. 3. Условие равновесного состояния частицы на поверхности жидкости

Fig. 3. The condition for the equilibrium of a particle on the surface of a liquid

$$
-978-
$$


Уравнение состояния для такой системы с учетом сил сопротивления при движении в вязкой жидкости в проекциях на ось 0Х, направленную вниз, имеет вид

$$
m \frac{\mathrm{d} v}{\mathrm{~d} t}=F_{m}-F_{\mathrm{A}}-F_{c},
$$

где $m$ - масса частицы; $\frac{\mathrm{d} v}{\mathrm{~d} t}$ - ускорение, приобретаемое частицей вследствие действия сил; $F_{\mathrm{A}}-$ сила Архимеда; $F_{c}$ - сила сопротивления.

Сила сопротивления или сила Стокса $[10,11]$ определяется по формуле

$$
F_{c}=4 \pi r v
$$

где $\pi \approx 3,14 ; r-$ радиус частицы; $v=\frac{\mathrm{d} x}{\mathrm{~d} t}-$ ее скорость.

Для упрощения понимания в модельных расчетах полагаем, что поверхность частицы близка к сферической. Скорость ее погружения определим из решения дифференциального уравнения (1). При условии, что в начальный момент времени сила сопротивления равна нулю, $v=0$, частица находится на поверхности жидкости. При решении также учитывалось, что объем газовой фазы в частице изменяется с течением времени и зависит от изменения краевого угла смачивания $\Theta$ с течением времени на границе раздела уголь-жидкость-газ и величины лапласовской силы смачивания. Численное решение (1) представлено на рис. 4 в виде зависимостей $v=\mathrm{f}(t)$.

Из графических зависимостей следует, что сначала частица некоторое время находится на поверхности жидкости - протекание процесса смачивания. Затем вследствие смачивания ее поверхности частица погружается на дно кюветы.

Существенной стороной в теории межфазного взаимодействия является изменение краевого угла смачивания с течением времени [7]. В данном случае этот процесс рассматриваем как гистерезис краевого угла смачивания, частично исследованного в работе. Показано существенное изменение краевого угла смачивания на границе раздела жидкость-уголь-газ $\Theta=f(t)$.

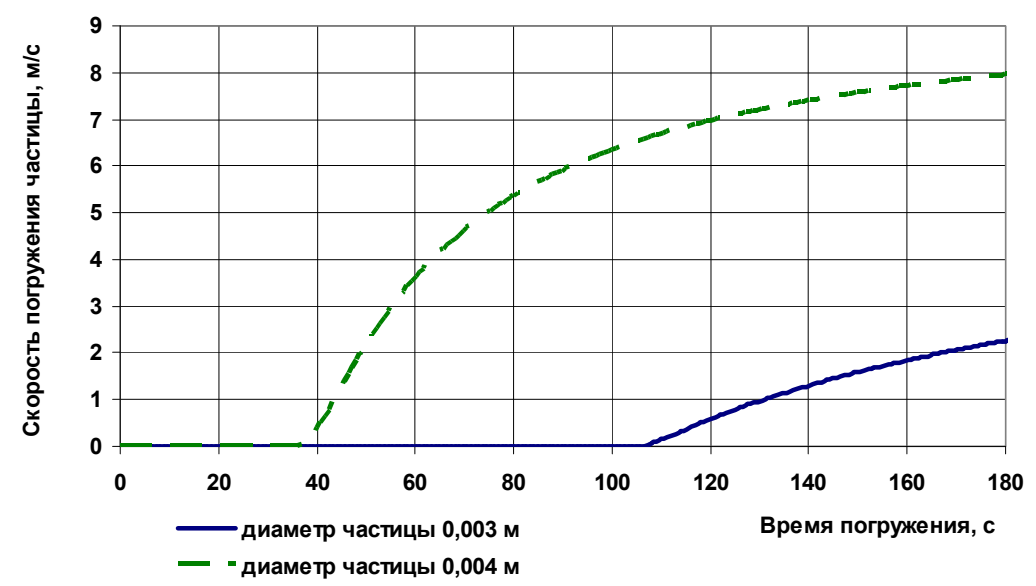

Рис. 4. Скорость погружения частицы в зависимости от размера

Fig. 4. Velocity of particle immersion depending on the size 
На рис. 5 приведен ряд зависимостей $\Theta=\mathrm{f}(t)$, полученных в опытах по результатам наблюдений за изменением краевого угла смачивания с течением времени.

Применение ПАВ приводит к изменению лиофильных свойств поверхности угля. Происходит более интенсивное вытеснение молекул воздуха с поверхности твердого тела, деполяризация поверхности, уменьшение краевого угла смачивания до 0 град. Поверхность становится гидрофильной. И в соответствии с описанным выше опытом частицы погружаются на дно кюветы с жидкостью в течение нескольких секунд.

При использовании ПАВ для повышения эффективности смачивания существенное значение имеет концентрация ПАВ в растворе, температура раствора, тип ПАВ. При оптимальной концентрации ПАВ и температуре жидкости смачивание протекает с максимальной скоростью.

С другой стороны, несоответствие между теоретической и экспериментальной зависимостью объясняется изменением физико-химической активности поверхности материалов в процессе пылеобразования. Процессы разрушения, механического деления на части и пылеобразования определяются физико-химической структурой угля и его свойствами в начальном состоянии.

Исследования по смачиваю пылеобразных частиц показывают, что в течение пылеобразования, образования частиц с малыми размерами свойства поверхностностей по физикохимической активности существенно отличаются от свойств тех же материалов, но больших размеров. Причем это различие для углей достигает значительно больших значений, чем для частиц других природных материалов.

При разрушении и пылеобразовании частиц, макромолекул угля, кристаллитов образуются радикальные комплексные соединения типа $-\mathrm{CH},-\mathrm{C}$. Взаимодействуя с кислородом воздуха, они окисляются до соединения $-\mathrm{COH},-\mathrm{CO}$ и др. В результате образуется гидрофобная поверхность с краевым углом смачивания, значительно отличающимся от первоначального и приближающегося к $130^{\circ}$ и более.

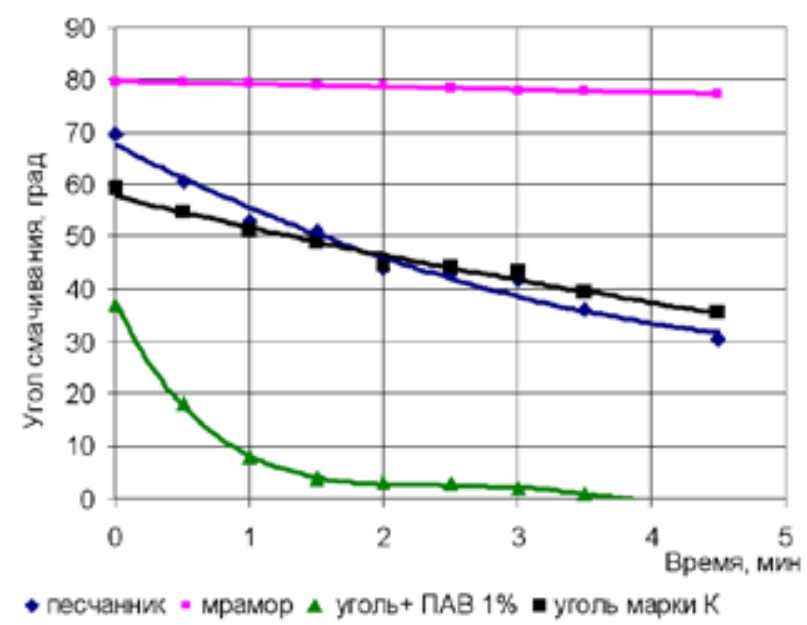

Рис. 5. Изменение краевого угла смачивания с течением времени

Fig. 5. Change of contact angle of wetting over time 
Таким образом, плотность образования гидрофобных центров на поверхности частицы зависит от размеров частиц. Уменьшение их размера приводит к увеличению плотности гидрофобных центров на поверхности частицы.

Исследования изменения упругих характеристик, модуля упругости в процессе дробления и образования мелких фракций показали существенную зависимость модуля упругости от размера фракции и существенное отличие от модуля упругости в целостном нетронутом угольном массиве [12]. Аналогичные работы, проведенные на фракциях песчаников, не демонстрировали таких зависимостей, что свидетельствует о значимом изменении физико-химических свойств угля в процессах дробления и разрушения до образования пылеобразных структурных частиц.

Сопоставление результатов экспериментальных исследований и модельных расчетов показывает, что в результате смачивания поверхности угля происходит значительное изменение объема газовой фазы в частице, вследствие чего существенно изменяется кажущая плотность вещества частицы. Для частиц менее 0,0005 мм углей средней стадии метаморфизма и более кажущая плотность угля менее 1000 кг/м³ , они имеют хорошую плавучесть на поверхности воды. Понижение кажущейся плотности частицы объясняется структурой макромолекулы и ее свойствами. В процессе разрушения, измельчения угля происходит разрушение межмолекулярных связей и в том числе частичное или полное разрушение макромолекул с образованием свободных радикалов, поляризации поверхности, активных гидрофобных молекулярных центров, что приводит к изменению физико-химических свойств угля и поверхностных свойств угля.

С другой стороны, опыты и наблюдения по смачиванию углей выявили, что уголь обладает анизотропной структурой. Величина краевого угла смачивания зависит от анизотропных свойств твердого тела. Из результатов исследований вытекает, что на поверхности аншлифа, полученного параллельно напластованию, краевой угол смачивания в зависимости от марки угля и физико-химических свойств изменяется от 20 до $55^{\circ}$. В то время как на поверхности аншлифа, полученного перпендикулярно напластованию, величина краевого угла смачивания по результатам наблюдений изменяется от 50 до $130^{\circ}$, т. е. поверхность проявляет гидрофобные свойства угля. Таким образом, в результате разрушения количество гидрофобных центров значительно увеличивается с уменьшением радиуса частицы, а процесс смачивания угля приобретает ярко выраженный анизотропный характер, зависящий от направления напластования.

В первом случае поверхность имеет более высокую твердость, меньшую проницаемость, радикальные поляризационные центры на поверхности отсутствуют, поверхность проявляет гидрофильные свойства. В этом направлении модуль упругости имеет меньшее значение, чем в направлении вдоль напластования.

Анализ результатов исследований показывает, что кроме действия лапласовских сил, сил сопротивления необходимо учитывать влияние процесса диффузии молекул воды через газовоздушную прослойку между твердой поверхностью частицы и жидкостью по закону диффузии Фика:

$$
\vec{j}_{m}=-D \operatorname{grad} C_{\rho},
$$

где $\vec{j}_{m}$ - плотность потока массы вещества; $D$ - коэффициент диффузии; $C_{p}-$ концентрация вещества $[13,14]$.

$$
-981-
$$


При взаимодействии частиц с жидкостью (раствором ПАВ) через воздушную прослойку между жидкостью и углем происходит диффузия молекул воды, вследствие чего влажность угля, смачиваемость увеличиваются, уменьшается краевой угол смачивания [3].

Диффузия молекул воды и адсорбции на поверхности раздела приводит к ее гидрофилизации, к уменьшению краевого угла смачивания. Причем с увеличением степени метаморфизма гидрофильные свойства изменяются. Коэффициент диффузии $D$ в соответствии с молекулярнокинетической теорией газов косвенно зависит от температуры. Увеличение температуры закономерно ведет к увеличению скорости диффузии и, соответственно, к изменению краевого угла смачивания. Повышение температуры вызывает рост коэффициента диффузии.

Молекулы воды, диффундирующие из газовоздушной среды, адсорбируются на поверхности твердой фазы. Вследствие чего изменяется адсорбционный потенциал поверхности твердого тела, из гидрофобной поверхности твердое тело становится гидрофильным [4].

Таким образом, в процессе смачивания частицы изменяется соотношение между действующими силами: силой Архимеда и силой тяжести, вследствие чего частица полностью смачивается и тонет, опускается на дно за некоторое время.

Время смачивания - это время, которое отражает скорость межфазных процессов, протекающих на поверхности частицы. Основной характеристикой, показывающей скорость протекания смачивания, будет время нахождения частицы на поверхности. По наблюдениям время смачивания много больше времени опускания на дно. Поэтому основная задача исследований сводится к нахождению времени смачивания, времени пребывания частицы на поверхности жидкости.

\section{Заключение}

Анализируя результаты экспериментальных исследований и модельных расчетов, можно прийти к следующим выводам. Средняя арифметическая скорость молекул определяется термодинамическими параметрами, в том числе температурой. Направленное движение молекул вследствие какого-либо физического воздействия приводит к конвекционному потоку, описываемому уравнением Навье-Стокса.

Постоянно протекающие геологические процессы, связанные с деформациями и разрушениями угольного пласта, в конечном итоге приводят к тому, что уголь становится потенциально выбросоопасным вследствие изменения структурных характеристик и, соответственно, физико-химической активности и сорбционных свойств. Нарушенный уголь выбросоопасных пластов имеет большее число гидрофобных составляющих в своей структуре и при гидрообработке требует применения растворов ПАВ, молекулы которого содержат группы -CH.

Из результатов наблюдений за поведением частиц на поверхности жидкости возможно в перспективе получение множества различных зависимостей и параметров, характеризующих межфазные взаимодействия в трехфазной системе: определение краевого угла смачивания, определение константы гистерезиса краевого угла смачивания, определение концентрации ПАВ в растворе и определение свойств угольных частиц (физико-химическая активность, активность жидкости или газа и др.).

Полученные результаты могут быть полезны при проектировании технологий обеспыливания подготовительных выработок, предварительном увлажнении угольного пласта, ис-

$$
-982-
$$


пользуемого для управления состоянием угольного массива путем применения растворов поверхностно-активных веществ, что позволяет повысить безопасность горных работ, эффективность отработки угольных пластов при использовании высокотехнологических комплексов в разработке угольных месторождений подземным способом.

\section{Список литературы}

[1] Чернов О.И., Розанцев Е.С. Подготовка шахтных полей с газовыбросоопасными пластами. М.: Недра, 1975. 287 с. [Chernov O.I., Rozantsev E.S. Preparation of mine fields with gazovybrosoopasnymi layers. Moscow, Nedra, 1975. 287 p. (in Russian)]

[2] Плотников Е.А., Дырдин В.В., Гвоздкова Т.Н. и др. Предотвращение динамических и газодинамических явлений при подземной разработке угольных пластов. Кемерово: Кузбассвузиздат, 2010. 165 с. [Plotnikov E.A., Dyirdin V.V., Gvozdkova T.N. Prevention and dynamic gas-dynamic phenomena in underground coal seams. Kemerovo, Kuzbassvuzizdat, 2010. 165 p. (in Russian)]

[3] Елкин И.С., Дырдин В.В., Михайлов В.Н. Повымение эффективности низконапорного увлажнения угольных пластов. Кемерово: Кузбассвузиздат, 2001. 100 c. [Elkin I.S., Dyirdin V.V., Mikhailov V.N. Improving the efficiency of low-pressure humidification of coal seams. Kemerovo, Kuzbassvuzizdat, 2001. 100 p. (in Russian)]

[4] Архипов В.А., Палеев Д.Ю., Патраков Ю.Ф., Усанина А.С. Определение краевого угла смачивания угольной поверхности. ФТПРПИ, 2011, 5, 22-27. [Arkhipov V.A., Paleev D.Y., Patrakov Y.F., Usanina A.S. Determination of the contact angle of the surface of coal. FTPRPI, 2011, 5, 22-27. (in Russian)]

[5] Горячев Б.Е., Николаев А.А., Ильина Е.Ю. Исследование кинетики флотации частиц с контролируемой степенью гидрофобности. ФТПРПИ, 2010, 1, 85-91. [Goryachev B.E., Nikolaev A.A., Ilyin E.Y. Kinetics of flotation of particles with a controlled degree of hydrophobicity. FTPRPI, 2010, 1, 85-91. (in Russian)]

[6] Назарова Л.А., Назаров Л.А., Полевщиков Г.Я., Родин Р.И. Определение коэффициента диффузии и содержания газа в угле на основе решения обратной задачи. ФТПРПИ, 2012, 5, 15-23. [Nazarova L.A., Nazarov L.A., Polevshchikov G.Y., Rodin R.I. Determination of the diffusion coefficient and the gas content of the coal on the basis of the inverse problem solution. FTPRPI, 2012, 5, 15-23. (in Russian)]

[7] Елкин И.С. Межфазные взаимодействия при увлажнении угольных пластов. Saarbrucken: LAP, 2014. 119 c. [Elkin I.S. Interfacial interaction when wet coal seams. Saarbrucken: LAP, 2014. 119 p. (in Russian)]

[8] Способ выбора поверхностно-активных веществ при увлажнении каменных углей по коэффиииенту пронииаемости. Патент на изобретение № 2533562. Патентообладатель: ФГБОУ ВПО «КузГТУ им. Т.Ф. Горбачева» (КузГТУ) (RU) - Заявка № 2012145884. Приоритет изобретения 16.07.2013 г. Зарегистрировано в Госреестре изобретений РФ 22.09.2014 г. [The method of selecting surfactants in wetting coals permeability coefficient. The patent for the invention № 2533562. The patent owner: VPO «KuzGTU them. T.F. Gorbachev» (KuzGTU) (RU). Registered in the State Register of Inventions of the Russian Federation of 09.22.2014 (in Russian)]

$$
-983-
$$


[9] Трубицына Д.А. Экспресс-метод оценки эффективности применения смачивателей для борьбы с пылью. Вестник ВостНИИ, 2010, 2, 202-206. [Trubitsyna D.A. Rapid method for evaluating the effectiveness of the use of wetting agents to control dust, Bulletin VostNII, 2010, 2, 202-206. (in Russian)]

[10]Ahmadi G. Hydrodynamic forces ME437/537 Clarkson Universite.

[11] Constantin P. Some open problems and research directions in the mathematical study of fluid dynamics, in Mathematics Unlimited. 2001 and Beyond, Springer Verlag, Berlin, 2001, 353-360.

[12]Иванов Б.М., Фейт Г.Н., Яновская М.Ф. Механические и физико-химические свойства углей выбросоопасных пластов. М.: Наука, 1979. [Ivanov B.M., Faith G.N., Yanovsky M.F. Mechanical and physico-chemical properties of coal seams outburst. Moscow, Nauka, 1979. 194 p. (in Russian)]

[13]Landau L., Lifchitz E. Fluid Mechanics. Pergamon Press, London. 1959.

[14]Ladyzhenskaya O. The Mathematical Theory of Viscous Incompressible Flows (2nd edition), Gordon and Breach, New York, 1969. 